\title{
Bücherschau.
}

Leitfaden zur chemischen Analyse in Anwendung bei Untersuchung chemischer Präparate. Ein Führer zur Bestimmung der Aechtheit und Güte und zum Nachweis von Verunreinigungen und Verfälschungen. Zum Gebrauch für Apotheker, Aerzte, Droguisten u. Chemiker, sowie für Studenten der Pharmacie und Medizin von Dr. Fr. Hoffmann, A potheker in New-York. D. Appleton \& Comp. 1873.

Unter diesem Gesammttitel haben wir ein Buch, welches Allen, denen es gewidmet ist, sehr willkommen sein wird. Ein Werk dieser Art hat lange in Amerika gefehlt und man kann mit Wahrheit sagen, duss der Verfasser diese Lücke in zufriedenstellender Weise ausgefüllt hat.

\section{A. Puttfarken.}

Jahresbericht der chem. Technologie für 1872 , v. R. Wagner. Leipzig bei $O$. Wigand. 1873.

Der Jahrgang 1872 dieses in technischen Kreisen anerkannten Werkes steht den fribheren in Mannigfaltigkeit des Inbalts und Uebersichtlicbkeit der Anordnung nicht nach. Wichtigere Processe sind im Original, andere in klaren, kurzen Auszïgen gegeben. Von besonderem Interesse sind die Berichte über einige metallurgische Verfahren: wie der Bessemerprocess, die Kupfergewinnung aus den Rückständen der Schwefelsäurefabriken, die Kiese verarbeiten, die Silberabtreibung aus Blei mittels Zink u. a. m. Die für das Beleuchtungswesen hochwichtige Erfindung von Klinkerfuess ist ausführlich behandelt und mit guten Abbildungen erläutert. Hier auf oine nähere Besprechung selbst nur einzelner Aibschnitte einzugehen, halten wir nicht für angezeigt, da das Thatsächliche dem Leserkreise schon bekannt sein wird, irgend ein Auszug daher überflüssig sein dürfte, während eine kritische Bcurtheilung der meisten in der Neuzeit in die Industrie eingefübrten Processe uberhaupt nur von einem speciell erfahrenen Chemiker versucht werden kann. $E$. Erhart.

Die technisch - chemischen Mittheilungen des Jahres 1872 bis 1873, ihrem wesentlichen Inhalte nach alphabetisch zusammengestellt von Dr. L. Elsner. Berlin, Verlag von Julius Springer. 1874.

Dieses Werk ist in seinen friheren Jahrgängen schon häufig im Archiv besprochen worden. Das dort Gesagte findet auch auf das vorliegende 2. Heft (der ganzen Reihe. XXII. Heft) Anwendung. Seit dem Erscheinen des ersten Heftes der ganzen Reihe ist im vorigen Jahre gerade ein Viertel-Säculum verflossen und hat der Herr Herausgeber, wie er in dem Vorwort mittheilt, bei dieser Veranlassung viele freundliche Zuschriften erhalten, die zugleich ein ehrendes Zeugniss für die Brauchbarkeit dieser technischen chemischen Mittheilungen ablegen. Das vorliegende Heft beginnt mit einer Zusammenstellung von Zeitschriften, theils wissenschaftlichen, theils chemisch-technischen Inhaltes (50 an Zahl), aus welchen die gebrachten Artikel im Auszug mitgetheilt werden, hierauf folgt ein reichhaltiges Verzeichniss neuer chemischer und chemisch-technischer Werke, ferner eire Vergleichung der jetzt in Deutschland neu

Arch, d. Pharm. L, Bds, 3. Hft. 
eingeführten Gewichte und Maasse mit den bis zum Jahr 1872 in Preussen in Gebrauch gewesenen, nebst Bemerkungen über deren Verhältniss zu französischen und englisclen Gewichten und Maassen. Sodann beginnen in alphabetischer Reihenfolge die Auszüge technisch-chemischer Abhandlungen, manche nur der Ueberscbrift nach, wobei indessen stets die Quellen bchufs weiterer Orientiringen gegeben sind.

Ihrem Inhalte nach wcrden die Artikel des vorliegenden Heftes unseren Lesern wohl grösstentheils bekannt sein, da sie ja 1-2 Jabre alt sind und ihre Runde durch viele technische und chemische Zeitschriften gemacht haben; es würde demnach überflüssig sein, bier näher auf den Inbalt einzugehen. Es genügt, dass derselbe in Ganzen sehr reichhaltig ist und die interessanteren Mittleilungen meist ziemlich ausführlich wiedergegrben sind, sodass der Besitz dieser "Mittheilungen" der vieler einzelner Journale ersetzen kann. Als Nachschlagebuch sei daher auch diese Fortsetzung des längst rühmlich bekannten Werkes bestens empfohlen.

J. Schnauss.

Dr. Otto Dammer. Kurzes chemisches Handwörterbuch zum Gebranche für Chemiker, Technik er, Aerzte, Pharmaceuten, Landwirthe, Lehrer und Freunde der Naturwissenschaften überhaupt. Berlin Verlag von Robert Opponheim. 1872. 8.

Die socben vorlicgenden 2., 3. und 4. Lieferung beginnen mit Anile und endigen nit Erdöl. Dem Programm gemäss werden alle Körper in gedrängtester Kürze abgehandelt, ohne dabei etwas Wichtiges oder Wissenswertbes auszulassen, so dass verschiedene Körper bloss mit einigen Zeilen bedacht sind. Grössere Räume sind dem Anthracen, Anthracit, Boden (Ackerboden), der Braunkohle, dem Brod, Campher, der Cellulose, dem Chinin, Chlor, der Chlor- und Chlorwasserstoffsäure, der Chromsäure, Citronensäure, dem Begriff Conserviren, dem Cyan, der Cyan- und Cyanwasserstoffsäure, Desinfection, Destillation, Diffusion, Dissociation, dem Dünger, den Eiern, Eisen, dem Eisenchlorid, Eisenferrocyanid und Eisenoxyd, den Eisenpräparaten, dem Eisensulfurete, Eiweiss, der Fiectricität, und Electrolyse gewidmet. Bei Anilin ist seine Gcschichte, sein Vorkommen, Darstellung und Verwendung angefuihrt. Unter Araeometer bespricht Verfasser in cingehendster Weise die verschiedenen Arten derselben und orläutert die Abweichungen von einander durch Tabellen; im Capitel Benzol wird dio Bildung verschiedener aromatischer Körper aus dem einfachen Kohlenwasserstoffe auf das Lehrreichste in gedrängter Kürze durchgeführt. Ferner folgt $\Delta$ rsenik, arsenige- und Arsensäure mit ibren Salzen. Abschnitt Asche bringt in Tabellenform 95 Aschenanalysen der verschiedensten Abstammung; Torf giebt 1-18\%, Braunkohle 1-40\%, Steinkohle $1-30 \%$, selten jedoch über $12 \%$ Asche. Capitel Athmung bespricht das Athmen der Thiere, wo Verfasser sagt: Die Aufnahme des Sauerstoffs ist nur zum kleinsten Theil die Folge des Druckes, unter welchem das Gas mit dem Blut in Berührung kommt, es ist vielmehr das Haemaglobin der Blutkörperchen, welches den Sauerstoff bindet, um ihn dann auf andere Körper zu übertragen. Im Grossen und Ganzen ist der thierische Stoffwechsel ein Oxydationsprocess in welchem die Blutkörperchen den Transport des Sauerstoffs ïbernehmen und so die 